\title{
Correction to: Incidence of Catastrophic Health Expenditure and Its Determinants in Cancer Patients: A Systematic Review and Meta-analysis
}

\author{
Leila Doshmangir $^{1,2}$ - Edris Hasanpoor ${ }^{3}$. Gerard Joseph Abou Jaoude ${ }^{4}$ Behzad Eshtiagh ${ }^{1}$. \\ Hassan Haghparast-Bidgoli ${ }^{4}$
}

Published online: 7 October 2021

(C) The Author(s), under exclusive licence to Springer Nature Switzerland AG 2021

\section{Correction to: Applied Health Economics and Health Policy https://doi.org/10.1007/s40258-021-00672-2}

In this article the affiliation of the co-author 'Dr. Hassan Haghparast-Bidgoli' was incorrectly given as

Department of Health Policy \& Management, Tabriz Health Services Management Research Center, School of Management \& Medical Informatics, Tabriz University of Medical Sciences, Tabriz, Iran

It should have been

The original article can be found online at https://doi.org/10.1007/ s40258-021-00672-2.

Edris Hasanpoor

edihasanpoor@gmail.com

Leila Doshmangir

Doshmangirl@tbzmed.ac.ir

Gerard Joseph Abou Jaoude gerard.jaoude.15@ucl.ac.uk

Behzad Eshtiagh

behzad_eshtiagh@yahoo.com

Hassan Haghparast-Bidgoli

h.haghparast-bidgoli@ucl.ac.uk

1 Department of Health Policy \& Management, Tabriz Health Services Management Research Center, School of Management \& Medical Informatics, Tabriz University of Medical Sciences, Tabriz, Iran

2 Social Determinants of Health Research Center, Health Management and Safety Promotion Research Institute, Tabriz University of Medical Sciences, Tabriz, Iran

3 Research Center for Evidence-Based Health Management, Maragheh University of Medical Sciences, Maragheh, Iran

4 Institute for Global Health, Center for Global Health Economics, University College London, London, UK
Institute for Global Health, Center for Global Health Economics, University College London, London, UK

And the affiliation of the co-author 'Behzad Eshtiagh' was incorrectly given as

Institute for Global Health, Center for Global Health Economics, University College London, London, UK

It should have been

Department of Health Policy \& Management, Tabriz Health Services Management Research Center, School of Management \& Medical Informatics, Tabriz University of Medical Sciences, Tabriz, Iran

The original article has been corrected. 